\title{
Increased expression of sonic hedgehog and altered methylation of its promoter region in gastric cancer and its related lesions
}

\author{
Li-Hui Wang,2,*, Yoon-La Choi ${ }^{3, *}$, Xuan-Yan Hua ${ }^{4,5}$, Young-Kee Shin ${ }^{1}$, Young-Jin Song ${ }^{6}$, \\ Sei-Jin Youn ${ }^{7}$, Hyo-Young Yun ${ }^{6}$, Seon-Mee Park ${ }^{7}$, Wun-Jae Kim ${ }^{8}$, Hee-Jin Kim ${ }^{9}$, \\ Jong-Sun Choi ${ }^{10}$ and Seok-Hyung Kim ${ }^{4}$
}

${ }^{1}$ Department of Pharmacy, Seoul National University, College of Pharmacy, Seoul, Korea; ${ }^{2}$ Department of Pharmacology, Shenyang Pharmaceutical University College of Pharmacy, Shenyang, China; ${ }^{3}$ Department of Pathology, Samsung Medical Center, Sungkyunkwan University School of Medicine, Seoul, Korea;

${ }^{4}$ Department of Pathology, Chungbuk National University College of Medicine, Cheongju, Chungbuk, Korea; ${ }^{5}$ Department of Pathology, Yanbian University College of medicine, Yanji, China; ${ }^{6}$ Department of Surgery, Chungbuk National University College of Medicine, Cheongju, Chungbuk, Korea; ${ }^{7}$ Department of Internal Medicine, Chungbuk National University College of Medicine, Cheongju, Chungbuk, Korea: ${ }^{8}$ Department of Urology, Chungbuk National University College of Medicine, Cheongju, Chungbuk, Korea; ${ }^{9}$ Department of Laboratory Medicine, Samsung Medical Center, Sungkyunkwan University School of Medicine, Seoul, Korea and ${ }^{10}$ Department of Pathology, Samsung Cheil Hospital, Sungkyunkwan University School of Medicine, Seoul, Korea

\begin{abstract}
The hedgehog (Hh) signaling pathway plays an important role in foregut development, and its activity is increased in various tumors, including those of the digestive tract. Our objective in the present study was to determine the pattern and extent of Sonic hedgehog (Shh) expression in gastric cancer and related lesions as well as the methylation status of its promoter region, in an attempt to clarify the regulatory mechanism of Shh expression. A total of 237 gastric cancers and related lesions (89 carcinomas, 22 high-grade dysplasia, 21 lowgrade dysplasia, 47 intestinal metaplasia, 38 chronic gastritis, and 20 normal epithelia) were subjected to immunohistochemical analysis with the Shh monoclonal antibody. The methylation status of Shh was determined by methylation-specific PCR (MS PCR), involving bisulfate treatment of DNA from 150 tissues followed by amplification using specific primer pairs designed by our group. Shh was completely absent in the upper part of normal gastric epithelia (gastric pit cells), and no significant differences were observed among the lower parts of normal epithelia, chronic gastritis, and intestinal metaplasia. However, Shh expression was significantly elevated in neoplastic lesions, such as carcinoma and high low-grade dysplasia, compared to nonneoplastic lesions. In carcinomas, Shh expression was associated with clinical stage, direct tumor invasion, and differentiation of tumor cells. Methylation of the Shh promoter region was frequent in normal gastric pit cells $(11 / 18,61.1 \%)$, but very rare in gastritis $(0 / 18)$, intestinal metaplasia $(0 / 19)$, dysplasia $(0 / 10)$, and carcinoma $(1 / 63,1.6 \%)$, and correlated significantly with expression $(P<0.001)$. Our results suggested that the increased and constitutive Shh expression is implicated in gastric carcinogenesis, and that promoter methylation may be an important regulatory mechanism of Shh expression.
\end{abstract}

Modern Pathology (2006) 19, 675-683. doi:10.1038/modpathol.3800573; published online 10 March 2006

Keywords: sonic hedgehog; gastric cancer; adenocarcinoma; dysplasia; intestinal metaplasia, methylation

Correspondence: Dr S-H Kim, MD, PhD, Department of Pathology, College of Medicine, Chungbuk National University, 62 Kaesindong, Cheongju, Chungbuk 361-763, South Korea.

E-mail: platoshkim@chungbuk.ac.kr

*These authors contributed equally to this study.

Received 27 July 2005; revised and accepted 27 January 2006; published online 10 March 2006
Hedgehog (Hh) comprises a family of secreted proteins that influence morphogenesis by providing positional information, such as the right-left axis, during development. Hh signaling is significantly involved in the development of different types of organs, from brain to limb, in a broad spectrum of animal species, including humans. In addition, the 
signaling pathway functions in the regulation of adult tissue stem cells during tissue regeneration after damage in adult. ${ }^{1,2}$

Three hedgehog (Hh) homologues have been identified in mammals, including Sonic hedghog (Shh), Indian hedgehog, and Desert hedgehog. In humans, the Hh signaling pathway is crucial for tissue development during the embryogenesis, such as the left-right asymmetry decision, anteriorposterior axis decision in limb pattern determination, and patterning of various organs, including brain, spinal cord, craniofacial structures, lung, teeth, eye, and hair. ${ }^{1,2}$ Shh is additionally essential for gastrointestinal tract development. Homozygous Shh knockout $\left(S h h^{-/-}\right)$mice show esophageal atresia/stenosis, tracheoesophageal fistula, and tracheal and lung anomalies, similar to the characteristics observed in humans with foregut defects. ${ }^{3}$ $S h h^{-1-}$ mice also display intestinal transformation of the stomach with overgrowth of the gastric epithelium, and duodenal stenosis caused by hyperplastic villi. ${ }^{4}$ Recent studies demonstrate that Shh is involved in the development of gastric gland and gastric epithelial differentiation in adults. ${ }^{5}$

Mutational activation of the Hh signaling pathway, either sporadic or in Gorlin's syndrome, is associated with tumorigenesis in a small subset of tissues, predominantly skin, cerebellum, and skeletal muscle. ${ }^{6,7}$ Gorlin's syndrome induced by a heterozygous mutation affecting the Patched gene, receptor for Shh, and is associated with various cancers, including basal cell carcinoma, medulloblastoma, and rhabdomyosarcoma. Furthermore, $\mathrm{Hh}$ signaling is implicated in carcinogenesis of the gastrointestinal tract. ${ }^{8,9}$

Here, we conducted a comprehensive immunohistochemical analysis of Shh expression at the protein level in cases of gastric cancer and its precancerous lesions, and determined the methylation status of the promoter region to elucidate the mechanisms of Shh upregulation in various lesions.

\section{Materials and methods}

\section{Patients, Tissue Samples, and Reagents}

We investigated 237 cases of gastric carcinoma and its related lesions obtained from the surgical pathology files maintained at the Department of Pathology of Chungbuk National University Hospital. The criteria for inclusion were as follows: histopathologic diagnosis of gastric lesions, and availability of clinical follow-up data as well as paraffin-embedded tissue specimens. All of the patients underwent surgical operations and none received either chemotherapy or radiotherapy before surgery. The selected cases consisted of 89 cases of adenocarcinoma, 22 cases of high-grade dysplasia, 21 cases of low-grade dysplasia, 47 cases of intestinal metaplasia, 38 cases of chronic gastritis, and 20 cases of normal gastric epithelium patients.
Slides were reviewed to analyze pathologic parameters, including tumor size, histological grading, depth of invasion, and the presence of nodal metastasis. The 89 gastric carcinomas (age range $=$ 30-76 years; average age $=58.75$ years; 32 female and 57 male) included 35 early cases (pTNM stage $\mathrm{I}=30$, pTNM stage $\mathrm{II}=5$ ) and 53 advanced cases (pTNM stage $\mathrm{III}=30$, pTNM stage $\mathrm{IV}=23$ ). Among these, $24(30.0 \%)$ were classified as welldifferentiated adenocarcinoma, $36(45.0 \%)$ as moderately differentiated, $20(25.0 \%)$ as poorly differentiated adenocarcinoma, and nine as signet ring cell type. TNM staging was assessed according to the system established by the American Joint Committee on Cancer (AJCC). ${ }^{10}$ In most cancer samples, intestinal metaplasia and atrophic gastritis were identified within close vicinity of the main tumor. Low- and high-grade dysplasia were differentiated on the basis of WHO guidelines. ${ }^{11}$ Two criteria were employed for differentiation between low- and high-grade dysplasia. Specifically, preservation of mucosal architecture and cellular atypism. Low-grade dysplasia (intraepithelial neoplasia, WHO terminology) is defined as a lesion with mild cellular atypia and minimal architectural distortion, including the preservation of tubular structures in their original form. In contrast, high-grade dysplasia is characterized by lesions with increasing architectural distortion and prominent cellular atypia. We examined three antral and 17 fundic normal gastric mucosa cases.

All archival materials were routinely fixed in $10 \%$ neutral-buffered formalin and embedded in paraffin. Sections $(4 \mu \mathrm{m})$ were prepared on silane-coated slides (Sigma, St Louis, MO, USA). Immunostaining kits were purchased from DAKO Inc. (Glostrup, Denmark).

\section{The Immunohistochemical Staining Procedure}

Tissue sections in microslides were deparaffinized with xylene, hydrated in serial dilutions of alcohol, and immersed in $3 \% \mathrm{H}_{2} \mathrm{O}_{2}$ to quench endogenous peroxidase activity. Next, sections were microwaved in $40 \mathrm{mM}$ borate buffer ( $\mathrm{pH}$ 8.3) supplemented with $1 \mathrm{mM}$ EDTA and $1 \mathrm{mM} \mathrm{NaCl}$ for $20 \mathrm{~min}$ for antigen retrieval. ${ }^{12,13}$ Avidin and biotin were consecutively applied to slides to prevent endogenous biotinrelated background staining. Sections were incubated with 1:50 diluted rabbit anti-Shh polyclonal antibody (sc-9024, Santa Cruz Biotech Inc., Santa Cruz, CA, USA) for $60 \mathrm{~min}$, followed by three successive rinses with washing buffer, and incubation with biotinylated goat anti-rabbit Abs (DAKO) for an additional $20 \mathrm{~min}$. After rinsing, tissue sections were incubated with HRP-conjugated streptavidin (DAKO) for $20 \mathrm{~min}$ at room temperature. Slides were washed, and the chromogen was developed for 5 min with liquid 3,3'-diaminobenzidine (DiNonA, Seoul, Korea). Next, slides were 
counterstained with Meyer's hematoxylin, dehydrated, and mounted with Canada balsam for examination. We used distilled water with $0.1 \%$ Tween-20 as a rinsing solution. ${ }^{14}$ Lymph node was employed as the positive control, since T lymphocytes and germinal center cells are positive for Shh, ${ }^{15-18}$ and smooth muscle as the negative control.

\section{Evaluation of Immunohistochemical Staining Data}

The scoring method by Sinicrope et a ${ }^{19}$ was applied for evaluating both the intensity of immunohistochemical staining and proportion of stained epithelial cells. Membranous and nuclear staining were independently analyzed. The staining intensity was subclassified as 1 (weak), 2 (moderate), and 3 (strong). Positive cells were quantified as a percentage of the total number of epithelial cells, and assigned to one of five categories: $0,<5 \% ; 1$, $5-25 \% ; 2,26-50 \%$; 3, 51-75\%; and $4,>75 \%$. The percentage of positivity of the tumor cells, and staining intensity were multiplied to generate the immunoreactive score (IS) for each tumor specimen. Each lesion was separately examined and scored by two pathologists (YLC and SHK), and cases with discrepant scores were discussed to obtain a consensus. IS values higher than 3 were classified as high expression.

\section{DNA Extraction}

DNA was prepared from 150 tissues of gastric carcinoma and related lesions. Sections $(10 \mu \mathrm{m})$ were dewaxed, H-E stained, and dried. Fields of interest were selected and microdissected under a microscope using a 27-gauge needle and micromanipulator. ${ }^{20}$ For each microtube $(1.5 \mathrm{ml})$, two sections of $20 \mu \mathrm{m}$ thickness were carefully collected to ensure equivalent amounts of tissue. Tissues and cells were autoclaved at $120^{\circ} \mathrm{C}$ for $20 \mathrm{~min}$ in $150 \mu \mathrm{l}$ of $5 \%$ Chelax 100 (Bio-Rad, Richmond, CA, USA) to extract DNA. Samples were centrifuged for $5 \mathrm{~min}$ at 10000 r.p.m., and the supernatant containing DNA was collected.

\section{Bisulfite Modification}

Methylation-specific-PCR (MS PCR) was performed according to the method of Olek et al. ${ }^{21}$ Briefly, $1 \mu \mathrm{g}$ DNA was diluted in $25 \mu \mathrm{l}$ of $\mathrm{dH}_{2} \mathrm{O}$ and heated at $95^{\circ} \mathrm{C}$ for $5 \mathrm{~min}$. Diluted DNA was mixed with $50 \mu \mathrm{l}$ of preheated and melted $2 \%$ low-temperature melting agarose gel (SeaPlaque, Cambrex, Rockland, ME, USA). Agarose beads were prepared by dropping $10 \mu \mathrm{l}$ of the mixture into parafilm cooled on ice. Agarose beads were placed in a microtube. Freshly prepared bisulfite solution $(1 \mathrm{ml})(3.5 \mathrm{M}$ sodium bisulfite, Sigma-Aldrich, Cat\#: 243973, $1 \mathrm{mM}$ hydroquinone, sigma, H-3660, $\mathrm{pH} 5.0$ ) was added to the microtube containing the agarose beads, and samples were incubated for $20 \mathrm{~h}$ at $50^{\circ} \mathrm{C}$ under a light-protected conditions. After washing twice with $1 \mathrm{ml}$ of $10 \mathrm{mM}$ Tris-HCl ( $\mathrm{pH}$ 8.0) and $10 \mathrm{mM}$ EDTA for $15 \mathrm{~min}$ each, the beads were desulfonated by washing three times with $1 \mathrm{ml}$ of $0.5 \mathrm{~N} \mathrm{NaOH}$ for $15 \mathrm{~min}$ each. After neutralization with $200 \mu \mathrm{l}$ of $1 \mathrm{~N}$ $\mathrm{HCl}$ for $5 \mathrm{~min}$, beads were washed twice with $1 \mathrm{ml}$ of $10 \mathrm{mM}$ Tris-HCl (pH 8.0) and $10 \mathrm{mM}$ EDTA, and sliced into several segments, one of which was directly utilized as a template for PCR.

\section{MS PCR for Shh}

The DNA methylation pattern in the CpG island of the Shh gene was determined by sodium bisulfite treatment and subsequent MS PCR. Primers for MS PCR were designed to encompass the CpG-rich area of the promoter region around the transcription initiation site (Figure 2). PCR primers were fully complementary to denatured DNA strands, and included at least two CG dinucleotides. The primer sequences are specified below: primary PCR, forward primer: $5^{\prime}$-GGG AGT GGT GGA GAG TTT TTT G-3', reverse primer: $5^{\prime}$-AAC TAA TAA CTT CCA AAC TAT CCC CA-3' for unmethylated DNA sequences; primary PCR, forward primer: 5'-GAG CGG TGG AGA GTT TTT CG-3', reverse primer: 5'-ACT AAT AAC TTC CGA ACT ATC CCC G-3' for methylated DNA sequences; nested PCR, forward primer: $5^{\prime}$-GGT GGA GAG TTT TTT GTA GTT GTG GT-3', reverse primer: $5^{\prime}$-AAA CTA TCC CCA TAC AAA TCC ATA CA-3' for unmethylated DNA sequences; and nested PCR, forward primer: 5'-AGA GTT TTT CGT AGT CGC GGC-3', reverse primer: 5'-ATC CCC GTA CGA ATC CGT ACG-3' for methylated DNA sequences. Unmethylated and methylated products were 179 and $169 \mathrm{bp}$ long, respectively. The conditions for the primary and nested MS PCR were as follows: denaturation at $95^{\circ} \mathrm{C}$ for $5 \mathrm{~min}, 40$ cycles of $94^{\circ} \mathrm{C}$ for $30 \mathrm{~s}, 64^{\circ} \mathrm{C}$ for $45 \mathrm{~s}, 72^{\circ} \mathrm{C}$ for $45 \mathrm{~s}$, and final extension at $72^{\circ} \mathrm{C}$ for $10 \mathrm{~min}$. The PCR mixture contained $1 \times$ buffer (TaKaRa, Japan) with $1.5 \mathrm{mM} \mathrm{MgCl}_{2}, 10 \mathrm{pmol}$ of each primer, $0.2 \mathrm{mM} \mathrm{dNTP}$ and $1 \mathrm{U}$ of Taq DNA polymerase (HotStart version, TaKaRa) and $100 \mathrm{ng}$ of bisulfite-modified DNA in a final volume of $50 \mu \mathrm{l}$. Normal human lymphocyte DNA methylated in vitro with SssI methylase (New England Biolab, Beverly, MA, USA) was employed as a positive control, while water blanks and PCR mixtures (without template) were used as negative controls in each assay. Following amplification, products were analyzed on $2 \%$ agarose gels stained with ethidium bromide, purified using a gel extraction kit (Qiagen, Valencia, CA, USA), and cloned into the pGEM-T vector (Promega, Woods Hollow Road Madison, WI, USA). Plasmid DNA was extracted from individual clones using a mini-prep kit (Qiagen) and sequenced (Cosmogenetech, Seoul, Korea). 


\section{Statistical Analysis}

Statistical analyses were conducted using Fisher's exact test, Pearson's $\chi^{2}$ test, ANOVA, MannWhitney test, Tukey's HSD, and Duncan's test as a post hoc analysis. $P$-values of $<0.05$ were regarded as statistically significant. All statistical analyses were performed using SPSS software (SPSS, Chicago, USA).

\section{Results}

\section{Shh Expression Patterns in Stomach Cancer and Its Precursor Lesions}

We analyzed Shh expression in gastric carcinomas, related neoplastic lesions (low-/high-grade dysplasia), intestinal metaplasia, chronic gastritis, and normal epithelium via immunohistochemistry.

Initially, the average intensity (mean of IS) of immunostaining was analyzed. In normal gastric epithelium, Shh expression was variable depending on the specific region of gastric mucosa. Expression was almost completely absent in the upper region of the normal gastric epithelium containing foveolar epithelia or pit cells, and apparently positive, albeit relatively weak, in the lower part containing mucus neck cells (gastric tissue stem cells) and glandular cells (Figures 1 and 3). In the lower region of the gastric epithelium, the staining intensity was highest in mucus neck cells, and decreased along glandular differentiation. The Shh expression was observed in all of fundic glands, but not in antral glands. In active chronic gastritis, Shh was expressed throughout most of the gastric epithelium, including the upper part containing the gastric pit (Figure 1, Table 1). We analyzed a total of 27 cases of complete intestinal metaplasia, and 20 cases of incomplete metaplasia. The average intensities (mean of IS) of Shh staining in complete and incomplete metaplasia were $2.46 \pm 2.02$ and $3.24 \pm 1.51$, respectively, with no significant differences $(P=0.173)$. However, Shh expression was influenced by the extent of regenerating activity and inflammation accompanying the intestinal metaplasia rather than the type of intestinal metaplasia itself. In many cases, metaplasia sometimes coincided with intense regeneration, whereas it did not from other regions in the same patient sample. In this case, the Shh levels were changed depending on the presence of the regenerating background, irrespective of the type of intestinal metaplasia. Generally, Shh expression was elevated in inflammation and regeneration-associated metaplasia, but was almost zero in simple intestinal metaplasia without such a regenerating activity (Figure 1). However, the overall average intensity in both chronic gastritis and intestinal metaplasia was similar to that in the lower part of normal epithelium (Table 1). Shh expression in neoplastic lesions including low-grade dysplasia and carcinoma, was significantly higher than that in metaplasia and normal epithelium $(P<0.05)$. However, no differences in expression were evident between these neoplastic lesions (Figure 1, Table 1). Notably, all Shh immunostainings were cytoplasmic.

We additionally analyzed Shh expression in terms of rate of high expression. In our experiments, cases with IS values higher than 3 were classified as 'high expression.' The results obtained regarding the rate of high expression were consistent with those from analysis of the average intensity of Shh immunostaining. Specifically, in normal epithelium, high Shh expression was rare, irrespective of the upper $(0 / 20,0 \%)$ or lower region $(4 / 20,20.0 \%)$. The rate was significantly increased in intestinal metaplasia $(24 / 47,51.1 \%)$ and various neoplastic lesions, including dysplasia $(29 / 43,67.4 \%)$, and carcinoma $(59 / 89,66.3 \%)$, compared to normal gastric epithelium and chronic gastritis $(11 / 38,28.9 \%)(P<0.05)$. However, no differences in the rate were evident among intestinal metaplasia and neoplastic lesions, including dysplasia and carcinoma.

\section{Correlation of Shh Expression with Clinicopathological Parameters in Gastric Cancer Patients}

The correlation of Shh expression with clinicopathological parameters in gastric cancer patients is depicted in Table 2. Shh expression was associated with direct tumor invasion, tumor grade, gross type, and clinical stage. The level of expression indicated by average intensity (mean of IS) was significantly higher in advanced gastric cancer than in early gastric cancer $(P<0.05)$. Moreover, expression was greatly enhanced in deeply invasive cases (T2-T4) and advanced stages (stage II-IV), compared with T1 and stage I ( $P<0.05$ for both comparisons). However, the level was decreased in Grade 3 cases (poorly differentiated) $(P<0.05)$, and the rate of high expression was also reduced in Grade 3 cases $(P<0.01)$. In addition, the rate was lower in diffusethan intestinal-type cancer $(P<0.01)$. Shh expression was not associated with the remaining clinicopathological parameters evaluated, including nodal metastasis, tumor size, sex, and age of patients.

Figure 1 Schematic representation of Shh expression and immunohistochemical staining of tissues in gastric carcinoma and its related lesions; CA, carcinoma; HD, high-grade dysplasia; LD, low-grade dysplasia; IM, intestinal metaplasia; CG, chronic gastritis; NL, normal epithelium. Expression in carcinoma and low-grade dysplasia is significantly higher than that in normal epithelium and intestinal metaplasia $(P<0.05)(\mathbf{a})$. Shh levels in individual cases of each disease entity are depicted in colors (b). Cytoplasmic staining of Shh was observed in most cases, except gastric pit of normal gastric epithelia (c). 
a

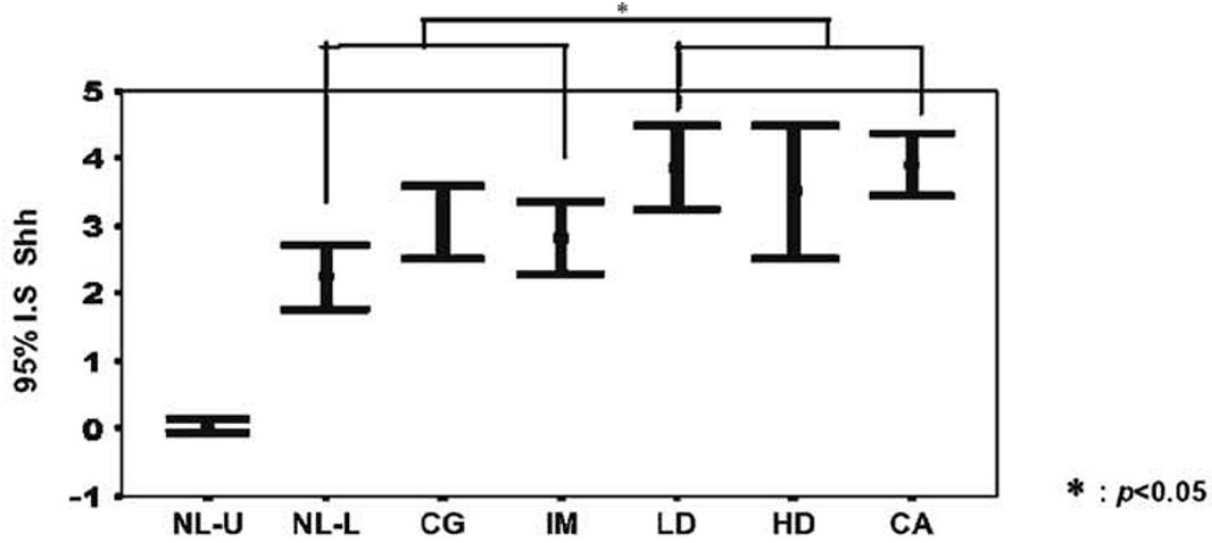

b
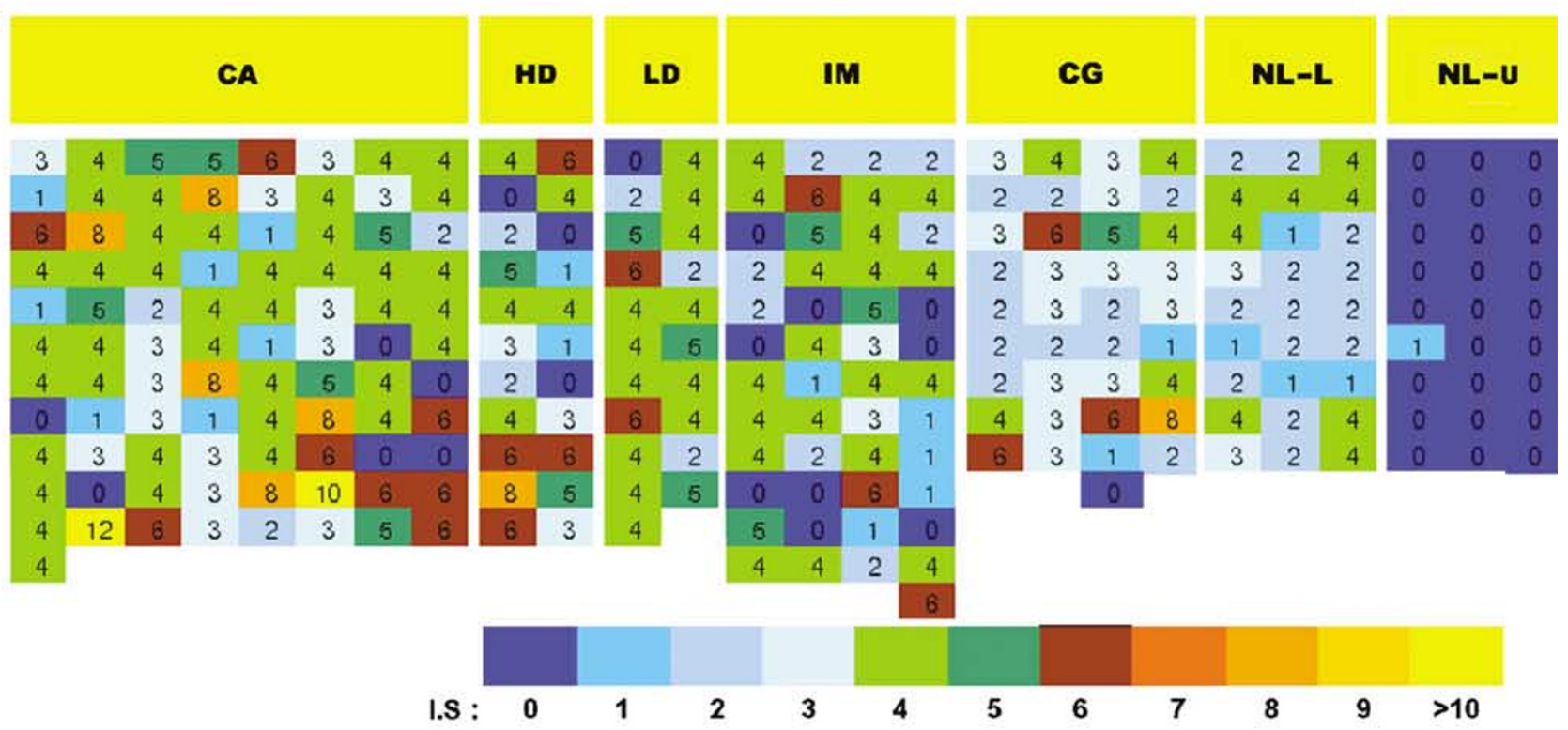

C

CA

HD

LD
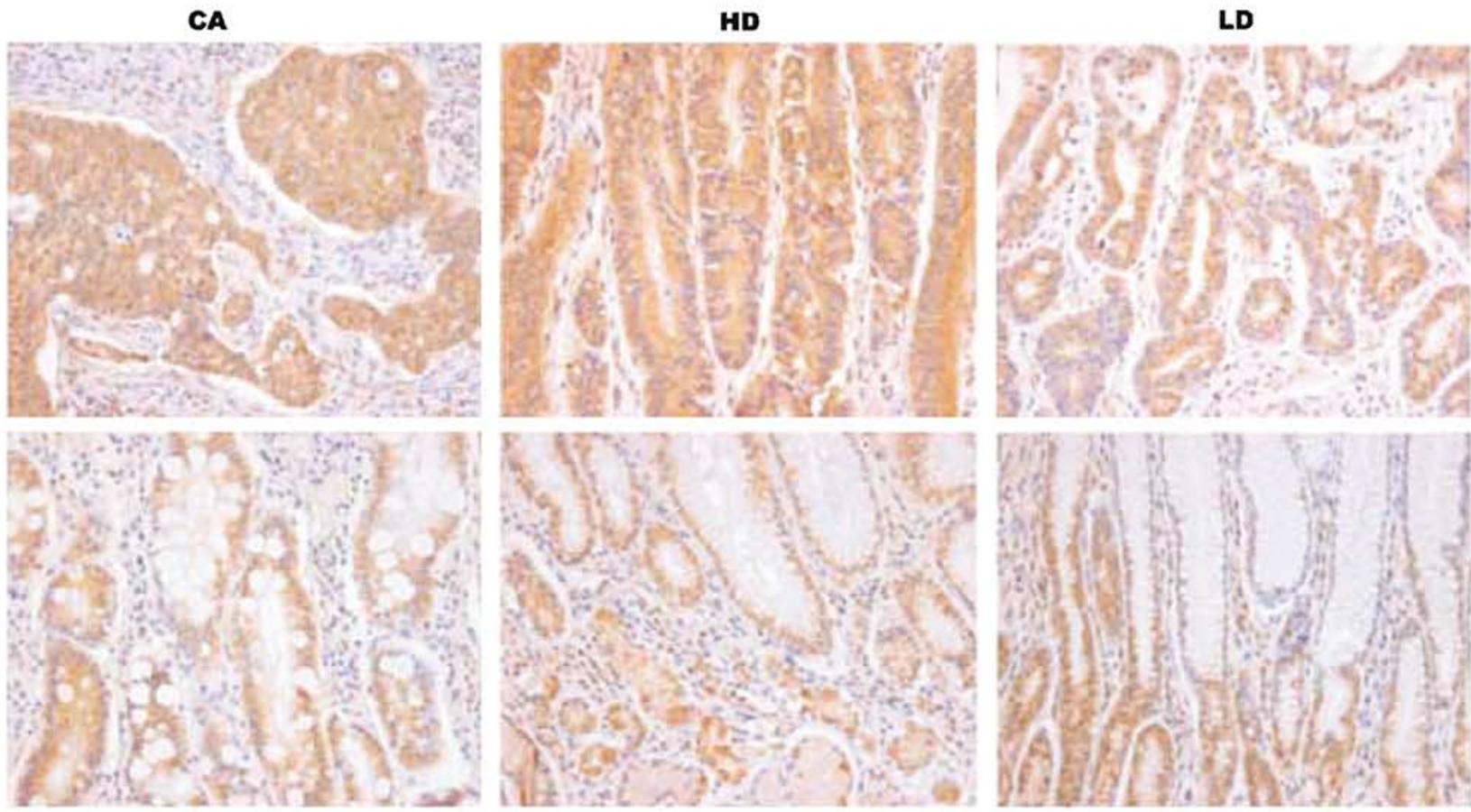

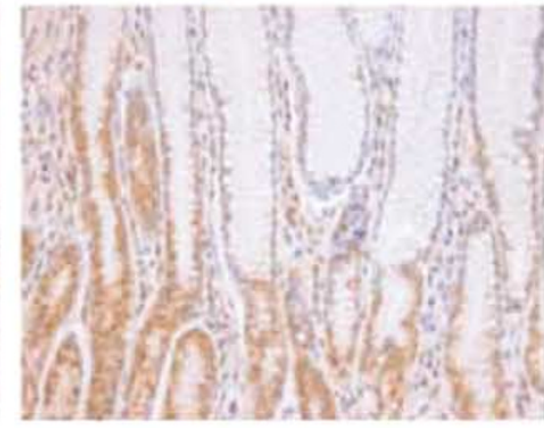

NL 
Table 1 Expression of Shh in gastric carcinoma and its related lesions

\begin{tabular}{lccc}
\hline Diagnosis & No. & $\begin{array}{c}\text { Mean of IS of } \\
\text { Shh immunostaining }\end{array}$ & P-value \\
\hline Carcinoma & 89 & $3.90 \pm 2.14$ & $<0.001$ \\
Dysplasia, high grade & 22 & $3.50 \pm 2.24$ & \\
Dysplasia, low grade & 21 & $3.86 \pm 1.39$ & \\
Intestinal metaplasia & 47 & $2.81 \pm 1.84$ & \\
Chronic gastritis & 38 & $3.05 \pm 1.63$ & \\
Normal (lower part) & 20 & $2.25 \pm 1.02$ & \\
Normal (upper part) & 20 & $0.01 \pm 0.22$ & \\
\hline
\end{tabular}

IS, immunoreactivity score.

Carcinoma vs intestinal metaplasia, chronic gastritis, normal epithelium: $P<0.05$.

Dysplasia (low grade) vs intestinal metaplasia, normal epithelium: $P<0.05$.

\section{Methylation Status of the Shh Promoter Region in Various Gastric Lesions}

The methylation status of the Shh promoter region was analyzed as one of the putative regulatory mechanisms of expression. The Shh promoter exists as a dense $\mathrm{CpG}$ island spanning about $300 \mathrm{bp}$ around the first exon (Figure 2). ${ }^{22}$ We performed methylation analysis of 150 samples including carcinomas and various precursor lesions, chronic gastritis, and normal epithelium (Table 3). In 117 of these cases, both methylation and immunohistochemical studies were performed (Table 4). The promoter region was partially methylated in only one of 63 carcinoma patients. No methylation was evident in dysplasia, intestinal metaplasia, chronic gastritis, and the

Table 2 Correlation of Shh expression with clinicopathological parameters in gastric cancer patients

\begin{tabular}{|c|c|c|c|c|c|c|}
\hline \multirow[t]{2}{*}{ Variable } & \multirow[t]{2}{*}{ No. } & \multicolumn{2}{|c|}{ Mean of IS } & \multicolumn{3}{|c|}{ Shh (+) No.\% } \\
\hline & & Cytoplasmic staining & $\mathrm{P}$-value & $\begin{array}{c}\text { Low or no expression } \\
(I . S<4)\end{array}$ & $\begin{array}{l}\text { High expression } \\
(I . S \geq 4)\end{array}$ & $\mathrm{P}$-value \\
\hline \multicolumn{7}{|l|}{ Age } \\
\hline$<60$ years & 38 & $4.03 \pm 2.47$ & \multirow[t]{2}{*}{0.630} & $14 / 38(36.8 \%)$ & $24 / 38(63.2 \%)$ & \multirow[t]{2}{*}{0.653} \\
\hline$\geq 60$ years & 51 & $3.80 \pm 1.88$ & & $16 / 51(31.4 \%)$ & $35 / 51(68.6 \%)$ & \\
\hline \multicolumn{7}{|l|}{ Sex } \\
\hline Male & 57 & $4.09 \pm 2.21$ & \multirow[t]{2}{*}{0.268} & $15 / 57(26.3 \%)$ & $42 / 57(73.7 \%)$ & \multirow[t]{2}{*}{0.063} \\
\hline Female & 32 & $3.56 \pm 2.00$ & & $15 / 32(46.9 \%)$ & $17 / 32(53.1 \%)$ & \\
\hline \multicolumn{7}{|l|}{ Tumor size } \\
\hline$<6.0 \mathrm{~cm}$ & 34 & $3.88 \pm 2.04$ & \multirow[t]{2}{*}{0.955} & $11 / 34(32.4 \%)$ & $23 / 34(67.6 \%)$ & \multirow[t]{2}{*}{1.000} \\
\hline$\geq 6.0 \mathrm{~cm}$ & 55 & $3.91 \pm 2.21$ & & $19 / 55(34.5 \%)$ & $36 / 55(65.5 \%)$ & \\
\hline \multicolumn{7}{|l|}{ Node metastasis } \\
\hline Negative & 30 & $3.57 \pm 2.08$ & \multirow[t]{2}{*}{0.333} & $12 / 30(40.0 \%)$ & $18 / 30(60.0 \%)$ & \multirow[t]{2}{*}{0.479} \\
\hline Positive & 58 & $4.03 \pm 2.17$ & & $18 / 58(31.0 \%)$ & $40 / 58(69.0 \%)$ & \\
\hline \multicolumn{7}{|l|}{ Gross type } \\
\hline AGC & 63 & $4.24 \pm 2.06$ & \multirow[t]{2}{*}{$<0.05$} & $18 / 63(28.6 \%)$ & $45 / 63(71.4 \%)$ & \multirow[t]{2}{*}{0.141} \\
\hline EGC & 26 & $3.08 \pm 2.13$ & & $12 / 26(46.2 \%)$ & $14 / 26(53.8 \%)$ & \\
\hline \multicolumn{7}{|l|}{ Lauren } \\
\hline Intestinal type & 58 & $4.41 \pm 1.71$ & \multirow[t]{2}{*}{$<0.05$} & $11 / 58(19.0 \%)$ & 47/58 (81.0\%) & \multirow{2}{*}{$<0.01$} \\
\hline Diffuse type & 21 & $3.57 \pm 2.56$ & & $12 / 21(57.1 \%)$ & $9 / 21(42.9 \%)$ & \\
\hline \multicolumn{7}{|l|}{ Grade } \\
\hline I & 24 & $4.42 \pm 1.95$ & \multirow[t]{3}{*}{$<0.05$} & $4 / 24(16.7 \%)$ & $20 / 24(83.3 \%)$ & \multirow[t]{3}{*}{$<0.001$} \\
\hline II & 36 & $4.61 \pm 1.93$ & & 6/36 (16.7\%) & $30 / 36(83.3 \%)$ & \\
\hline III & 20 & $3.10 \pm 1.71$ & & $13 / 20(65.0 \%)$ & $7 / 20(35.0 \%)$ & \\
\hline \multicolumn{7}{|l|}{ Tumor invasion } \\
\hline $\mathrm{I}$ & 25 & $3.04 \pm 2.17$ & \multirow[t]{4}{*}{$<0.05^{\mathrm{a}}$} & $12 / 25(48.0 \%)$ & $13 / 25(52.0 \%)$ & \multirow[t]{4}{*}{0.229} \\
\hline II & 19 & $4.95 \pm 2.66$ & & 4/19 (21.1\%) & 15/19 (78.9\%) & \\
\hline III & 31 & $3.81 \pm 1.66$ & & $11 / 31(35.5 \%)$ & $20 / 31(64.5 \%)$ & \\
\hline IV & 13 & $4.08 \pm 1.71$ & & $3 / 13(23.1 \%)$ & $10 / 13(76.9 \%)$ & \\
\hline \multicolumn{7}{|l|}{ pTNM staging } \\
\hline I & 30 & $3.20 \pm 2.02$ & $0.07^{\mathrm{b}}$ & 13/30 (43.3\%) & 17/30 (56.7\%) & 0.429 \\
\hline II & 5 & $5.60 \pm 2.51$ & & $2 / 5(40.0 \%)$ & $3 / 5(60.0 \%)$ & \\
\hline III & 30 & $4.20 \pm 1.97$ & & $7 / 30(23.3 \%)$ & $23 / 30(76.7 \%)$ & \\
\hline IV & 23 & $3.96 \pm 2.23$ & & $8 / 23(34.8 \%)$ & $15 / 23(65.2 \%)$ & \\
\hline
\end{tabular}

${ }^{\mathrm{a}} \mathrm{T} 1$ vs $\mathrm{T} 2+\mathrm{T} 3+\mathrm{T} 4: P<0.05$.

${ }^{\mathrm{b}}$ Stage I vs II+III+IV: $P<0.05$. 
lower part of normal gastric epithelium. However, methylation was observed in 11/18 (61\%) samples of the upper part of gastric epithelium (Table 3, Figure 3). We confirmed the methylation status in selected representative cases by sequencing after
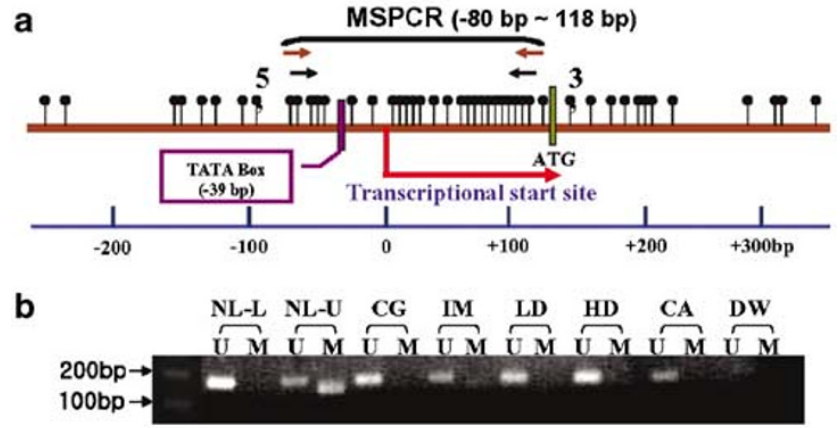

\section{C}

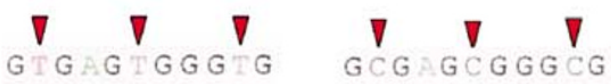

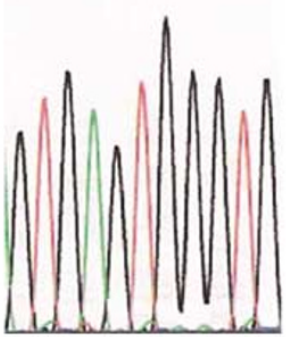

UM

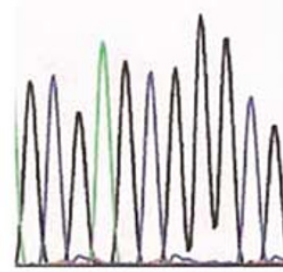

M
Figure 2 The region between -200 and +300 of the Shh $5^{\prime}$ flanking (a). The brown arrows signify the locations of the primers for primary PCR, and the black arrows specify those for the nested PCR. Black circles, CpGs. Methylation-specific PCR (MS PCR) of the Shh promoter in gastric carcinoma and its related lesions (b) and the representative sequences of MS PCR products (c). Primer sets used for amplification are classified as 'unmethylated' (U) or 'methylated' (M). Methylation was observed almost exclusively in the upper part of normal mucosa (NL-U) containing gastric pit (a). Abbreviations: CA, carcinoma; HD, high-grade dysplasia; LD, low-grade dysplasia; IM, intestinal metaplasia; CG, chronic gastritis; NL-U, upper part of normal epithelium; NL-L; lower part of normal epithelium. The sequence from unmethylated DNA samples contains a $\mathrm{C}$ to $\mathrm{T}$ conversion (arrows), whereas the corresponding sequence in methylated cases retains C (arrows), indicating methylation of these sites.

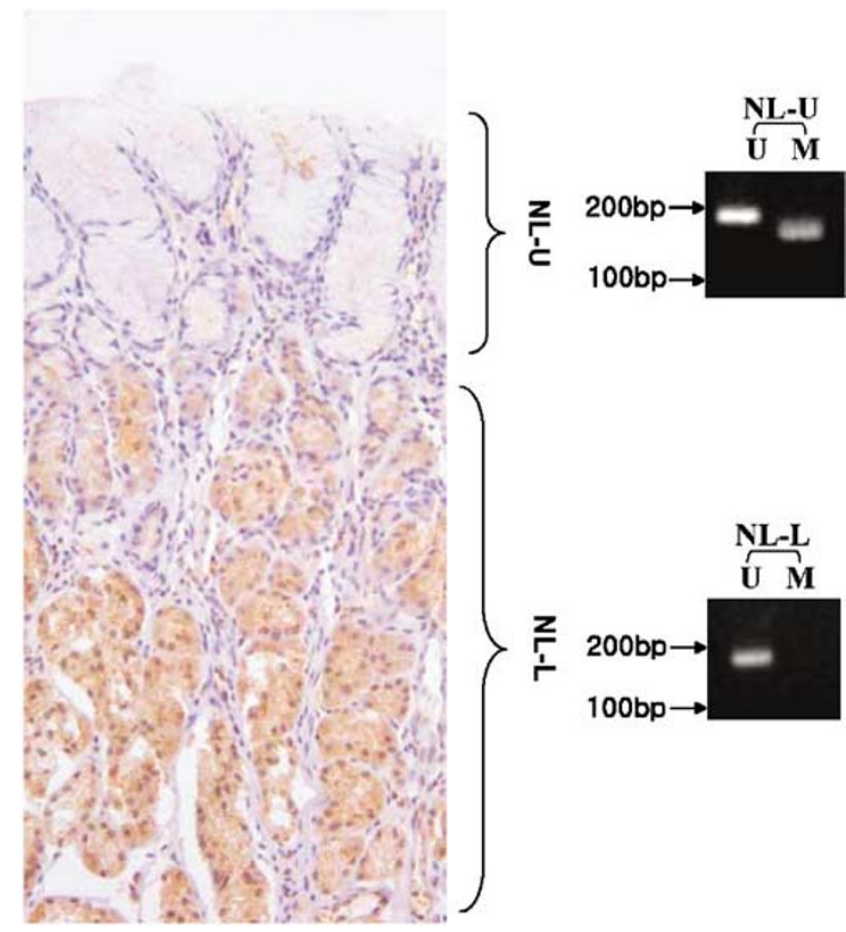

Figure 3 The methylation status of normal gastric epitheium. In the upper part of the normal epithelium (NL-U) containing gastric pit, Shh expression was absent and the promoter was methylated. However, in the lower part (NL-L) containing mucus neck cells (tissue stem cells) and glandular cells, Shh was expressed, and its promoter remained unmethylated.

Table 4 Frequency of protein loss in gastric carcinomas with Shh promoter methylation

\begin{tabular}{lrrr}
\hline Shh methylation & \multicolumn{2}{c}{ Shh immunostaining } & Total \\
\cline { 2 - 3 } & \multicolumn{1}{c}{+} & \\
\hline Negative & 3 & + & \\
Positive & 9 & 104 & 107 \\
Total & 12 & 1 & 10 \\
& & 105 & 117 \\
\hline
\end{tabular}

$P<0.001$ 
cloning (Figure 2). In addition, strong correlation was evident between Shh expression and promoter methylation (Table 4). Specifically, promoter methylation was observed in nine out of 12 cases $(75 \%)$ displaying no Shh expression, and in only one of $105(1 \%)$ cases that were positive for Shh (Table 4).

\section{Discussion}

In this study, we characterize the Shh expression patterns, in stomach cancer and related lesions by immunohistochemistry. We additionally determine the relationship between Shh expression and clinicopathological parameters in gastric cancer patients. And a close correlation between the methylation status of the promoter region and Shh expression was observed.

Our results show that the Shh expression is increased in two stages during the whole metaplasia-dysplasia-early carcinoma-advanced carcinoma sequence of stomach cancer. The first increase occurs at the metaplasia-dysplasia transition, and the second boost occurs during the progression from early to advanced gastric carcinoma. The implications of these findings remain to be clarified. However, the significance of high expression in these neoplastic lesions is evident from several previous studies on the role of unregulated and persistent Shh activation in the connection between chronic tissue injury and cancer. ${ }^{8,23,24}$ Persistent Shh activation related to chronic injury is required for tumorigenesis, instead of the transient activation induced by acute injury. This continuous Shh activation induces expansion in a pool of activated stem cells. Consequently, the total number of activated stem cells is greatly elevated over time, increasing the probability that an oncogenic event will trap stem cells in the activated state. The Hh signaling pathway plays a central role in directing embryonic pattern formation, and also functions postembryonically in stem cell renewal and tissue repair and regeneration. ${ }^{23}$ In gastric cancer, aberrant Hh signaling is mediated by abnormal Shh levels, accompanied by reception of the signal within the same tumor cells. ${ }^{8}$ Blockers of the Hh pathway inhibit the growth of gastric carcinoma cell lines. ${ }^{8}$ The activated Hh pathway has been reported in other organs. ${ }^{23}$ Familial mutations that facilitate $\mathrm{Hh}$ and WNT pathway activation have been associated with increased incidence of cancers in the brain, skin, skeletal muscle, liver, and colon in both humans and mice. Additional studies in which pathway activities are antagonized by treatment with pharmacological agents, antibodies that bind and block the ligand action, or overexpression of negative regulatory components should further confirm the ongoing requirement for pathway activity in the growth of other cancer types including small cell lung cancer and carcinoma of esophagus, stomach, pancreas, biliary tract, and prostate. $^{23}$

Our results on the distribution of Shh expression in normal gastric epithelium are consistent with data from a previous report. Shh was almost completely absent in foveolar epithelium (gastric pit cells), as shown by Van den Brink et al. ${ }^{25}$ Moreover, Shh expression was rare and very weak in the antral gland, and relatively high in the fundic gland, with a few exceptions. Data on the methylation status of the promoter region support these results. Interestingly, in contrast to the findings under normal conditions without inflammation, Shh expression was expanded along the gastric pit (foveolar epithelium) with more intense inflammatory activity, particularly in chronic atrophic gastritis. In intestinal metaplasia, Shh expression was characteristically polarized between inflammation and regeneration-associated intestinal metaplasia and simple metaplasia without background inflammation and regeneration. In cases of simple intestinal metaplasia, Shh expression was absent or greatly reduced. However, in inflammation and regeneration-associated metaplasia with overproliferation of metaplastic glands, Shh expression was significantly increased in proportion to the intensity of proliferative activity. While the significance of this observation is still elusive, the reason is inferred from the dual function of Shh, that is, one, to increase the stem cell number during regeneration, and the other, to provide a polarizing signal in asymmetrical differentiation of gastric mucosa to the gastric pit and glandular components. ${ }^{8,23,24}$ Therefore, Shh expression is absent in simple metaplasia where asymmetrical differentiation was lost, but increased in inflammation and regeneration-associated metaplasia related to the intense proliferation of tissue stem cells.

Methylation of Shh promoter was frequent in normal gastric pit (foveolar epithelium), and a strong correlation was evident between the methylation status of the Shh promoter and Shh expression. DNA methylation in the promoter regions of many genes is associated with the regulation of gene expression. It results in transcriptional silencing of the gene, either through a direct effect or via changes in the chromatin conformation that inhibits transcription. To our knowledge, this is the first report on the methylation status of the Shh promoter. We demonstrate that the methylation in the Shh promoter is absent or vary rare in chronic active gastritis, intestinal metaplasia, low-/high-grade dysplasia, and carcinoma, further supporting data from the immunohistochemical study. The results collectively suggest that the promoter methylation of the Shh gene is a major regulatory mechanism of expression.

In summary, increased and persistent Shh expression may be implicated in gastric carcinogenesis, and promoter methylation is possibly an important regulatory mechanism of Shh expression. While the 
functional and clinical significance of the Hh pathway remains to be elucidated, it is suggested that Shh is a linker between chronic tissue injury and cancer, and thus a potential diagnostic biomarker and therapeutic target.

\section{Acknowledgement}

This work was supported by the Chungbuk National University Research grant.

\section{References}

1 McMahon AP. More surprise in the hedgehog signaling pathway. Cell 2000;10:185-188.

2 Wetmore C. Sonic hedgehog in normal and neoplastic proliferation: insight gained from human tumors and animal models. Curr Opin Genet Dev 2003;13:34-42.

3 Litingtung Y, Lei L, Westphal $\mathrm{H}$, et al. Sonic hedgehog is essential to foregut development. Nat Genet 1998; 20:58-61.

4 Ramalho-Santos M, Melton DA, McMahon AP. Hedgehog signaling regulate multiple aspects of gastrointestinal development. Development 2000;127:2763-2772.

5 Van den Brink GR, Hardwick JCH, Tytgat GNJ, et al. Sonic hedgehog regulates gastric gland morphogenesis in man and mouse. Gastroenterology 2001;121:317-328.

6 Bale AE, Yu KP. The hedgehog pathway and basal cell carcinomas. Hum Mol Genet 2001;10:757-762.

7 Wechsler-Reya R, Scott MP. The developmental biology of brain tumors. Annu Rev Neurosci 2001;24: 385-428.

8 Berman DM, Karhadkar SS, Maltra A, et al. Widespread requirement for hedgehog ligand stimulation in growth of digestive tract tumours. Nature 2003;425: 846-851.

9 Thayer SP, di Magliano MP, Heiser PW, et al. Hedgehog is an early and late mediator of pancreatic cancer tumorigenesis. Nature 2003;425:851-856.

10 Greene FL, Page DL, Fleming ID, et al. Morrow M American Joint Committee on Cancer Staging Manual, 6th edn. Springer: Berlin 2002, pp 139-144.

11 Hamilton AR, Aaltonen LA (eds). Pathology \& genetics Tumours of the digestive system, WHO Classification of Tumours. IARC press: Lyon, 2000, pp 46-48.

$12 \mathrm{Kim}$ SH, Kook MC, Song HG. Optimal conditions for the retrieval of CD4 and CD8 antigens in formalin- fixed, paraffin-embedded tissues. J Mol Histol 2004; 35:403-408.

$13 \mathrm{Kim}$ SH, Kook MC, Shin YK, et al. Evaluation of antigen retrieval buffer systems. J Mol Histol 2004; 35:409-416.

$14 \mathrm{Kim}$ SH, Shin YK, Lee KM, et al. An improved protocol of biotinylated tyramine-based immunohistochemistry minimizing nonspecific background staining. J Histochem Cytochem 2003;51:129-132.

15 Lowrey JA, Stewart GA, Lindey S, et al. Sonic hedgehog promotes cell cycle progression in activated peripheral CD4(+) T lymphocytes. J Immunol 2002; 169:1869-1875.

16 Stewart GA, Lowrey JA, Wakelin SJ, et al. Sonic hedgehog signaling modulates activation of and cytokine production by human peripheral CD4+ T cells. J Immunol 2002;169:5451-5457.

17 Stewart GA, Hoyne GF, Ahmad SA, et al. Expression of the developmental Sonic hedgehog (Shh) signalling pathway is up-regulated in chronic lung fibrosis and the Shh receptor patched 1 is present in circulating T lymphocytes. J Pathol 2003;199:488-495.

18 Sacedon R, Diez B, Nunez V, et al. Sonic hedgehog is produced by follicular dendritic cells and protects germinal center B cells from apoptosis. J Immunol 2005;174:1456-1461.

19 Sinicrope FA, Ruan SB, Cleary KB, et al. bcl-2 and p53 oncoprotein expression during colorectal tumorigenesis. Cancer Res 1995;55:237-241.

20 Lee JY, Dong SM, Kim SY, et al. A simple, precise and economical microdissection technique for analysis of genomic DNA from archival tissue sections. Virchows Arch 1998;433:305-309.

21 Olek A, Oswald J, Walter J. A modified and improved method for bisulphite based cytosine methylation analysis. Nucl Acids Res 1996;24:5064-5066.

22 Kitazawa S, Kitazawa R, Tamada H, et al. Promoter structure of human sonic hedgehog gene. Biochim Biophys Acta 1998;1443:358-363.

23 Beachy PA, Karhadkar SS, Berman DM. Tissue repair and stem cell renewal in carcinogenesis. Nature 2004; 432:324-331.

24 Watkins DN, Peacock CD. Hedgehog signaling in foregut malignancy. Biochem Parmacol 2004;68:10551060.

25 Van den Brink GR, Hardwick JCH, Nielson C, et al. Sonic hedgehog expression correlates with fundic gland differentiation in the adult gastrointestinal tract. Gut 2002;51:628-633. 